\title{
Osteoglycin knockdown promotes vascular smooth muscle cell proliferation and migration in aortic dissection via the VEGF/VEGFR2 axis
}

\author{
ZANXIN WANG ${ }^{1,2}$, XIANMIAN ZHUANG ${ }^{1}$, BAILANG CHEN $^{1}$ and MINXIN WEI ${ }^{1,2}$ \\ ${ }^{1}$ Department of Cardiac Surgery, Fuwai Hospital Chinese Academy of Medical Sciences Shenzhen, \\ Shenzhen, Guangdong 518057; ${ }^{2}$ Department of Cardiac Surgery, The University of Hong Kong-Shenzhen Hospital, \\ Shenzhen, Guangdong 518053, P.R. China
}

Received May 13, 2020; Accepted October 23, 2020

DOI: $10.3892 / \mathrm{mmr} .2020 .11703$

\begin{abstract}
Extracellular matrix (ECM) proteins serve a major role in the pathogenesis of aortic dissection (AD). The aim of the present study was to investigate the effect of osteoglycin (OGN), an ECM proteoglycan, on aortic dissection (AD), as well as the underlying mechanism. Thoracic aortic tissues from 20 patients with AD and healthy thoracic aortic tissue from 5 patients undergoing coronary artery bypass grafting were collected to detect OGN expression levels. Following OGN knockdown in rat aortic smooth muscle cells, cell proliferation was detected by performing Cell Counting Kit-8 and BrdU assays, cell migration was assessed by performing the wound healing assay, cell invasion was detected by performing the Transwell assay, and VEGFR/AKT signaling pathway-related protein expression levels were measured via western blotting. The results demonstrated that OGN expression was significantly downregulated in patients with AD compared with healthy controls. Compared with the si-negative control (NC) group, OGN knockdown promoted RASMC proliferation and migration. Compared with the si-NC group, OGN knockdown also significantly enhanced the phosphorylation of the downstream signaling molecules of VEGFR, including AKT and ERK1/2, in VEGF-stimulated RASMCs. Collectively, the present study indicated that OGN knockdown facilitated RASMC proliferation and migration by activating AKT and ERK1/2 signaling. Therefore, OGN may serve as a novel therapeutic target for AD.
\end{abstract}

Correspondence to: Dr Minxin Wei, Department of Cardiac Surgery, Fuwai Hospital Chinese Academy of Medical Sciences Shenzhen, 12 Langshan Road, Nanshan, Shenzhen, Guangdong 518057, P.R. China E-mail:weimx@hku-szh.org

Key words: aortic dissection, osteoglycin, VEGF, VEGFR2, angiogenesis

\section{Introduction}

Aortic dissection (AD) is a major complication of thoracic aortic disease, which is initiated by tears in the aortic intima and media. The tears allow blood to enter into the media and separate the medial layer along the long axis of the aorta, thus leading to the formation of a false lumen $(1,2)$. Multiple factors, including poorly controlled hypertension, older age, male gender, smoking, genetic conditions, pre-existing aortic diseases, aortic instrumentation or surgery, and immune/inflammatory diseases, are associated with an increased risk of $\mathrm{AD}$ (3-5). Although $\mathrm{AD}$ is an uncommon disease with an estimated annual incidence of 5-30 cases per million individuals (6), the disease displays high mortality and is ranked among the most lethal vascular diseases worldwide (7-9). Despite advances in therapeutic strategies, the cellular and molecular mechanisms underlying AD are not completely understood and require further investigation.

$\mathrm{AD}$ is pathologically characterized by the degeneration of the aortic media, which includes depletion of smooth muscle cells, destruction of elastic fibers and disruption of the extracellular matrix (ECM) network $(10,11)$. ECM components in the aortic wall not only provide structural support for vascular cells, but also integrate extracellular signals and modulate cellular responses (12). Alterations in ECM components serve critical roles in the pathogenesis of AD (13). Increasing evidence has suggested that an increase in the expression of proteoglycans, a major group of nonfibrillar ECM components, is a crucial event in AD and is closely associated with the degeneration of the aortic media $(12,14)$. Therefore, exploring the expression profiles of proteoglycans and their functional effects on medial smooth muscle cells is important for understanding the development of AD.

In our previous study, 99 aortic tissue samples from patients with AD were collected, and the genomic profiles were analyzed (15). A total of 3,425,873 SNPs, 685,245 insertion-deletions and 1,177 copy number variations were identified. By performing disease correlation analysis, 20 candidate genes were identified. A number of identified genes, such as myosin heavy chain 11 , fibrillin 1 and actin $\alpha 2$, smooth muscle, were consistent with previous studies (16-18), whereas, 
to the best of our knowledge, MAX dimerization protein MLX, DAB2 interacting protein, E1A binding protein p300, zinc finger FYVE-type containing 9, PML nuclear body scaffold, protein kinase $C-\delta$ and osteoglycin (OGN) were identified as AD-associated genes for the first time in our previous study.

OGN, which belongs to cluster III of the small leucine-rich proteoglycans (SLRP), is an ECM component that modulates various biological processes, including cell proliferation, inflammation and collagen fibrillogenesis (19-21). OGN is involved in numerous pathological conditions, including bone disease, eye disease, neurological damage and cancer (22). Plasma OGN expression levels are lower in patients with coronary artery disease with complex atherosclerotic lesions compared with patients with coronary artery disease without lesions (23). Proteomic analysis has demonstrated decreased OGN expression levels in calcified abdominal aortic aneurysm tissues compared with healthy adjacent aortic tissues (24). Although emerging evidence has revealed the effects of OGN on vascular diseases, the exact role of $\mathrm{OGN}$ in $\mathrm{AD}$ formation requires further investigation.

The present study aimed to investigate the effect of OGN, an ECM proteoglycan, on AD, as well as the underlying mechanism. Therefore, OGN expression profiles in thoracic aortic tissues from patients with $\mathrm{AD}$ and healthy thoracic aortic tissues from control subjects were determined. The effect of OGN on cellular proliferation and migration was determined in cultured rat aortic smooth muscle cells (RASMCs). RASMCs are typically used to study alterations in cellular and molecular biology during the progression of vascular disease under the influence of internal and external factors, and are a good model for studying mechanisms in vitro (25). The present study investigated the possible mechanisms underlying OGN in the pathological process of AD.

\section{Materials and methods}

Cell culture. RASMCs (Procell Life Science \& Technology Co., Ltd.) were cultured in DMEM (Procell Life Science \& Technology Co., Ltd.) supplemented with 10\% FBS (Gibco; Thermo Fisher Scientific, Inc.) and 1\% penicillin/streptomycin in a humidified incubator at $37^{\circ} \mathrm{C}$ with $5 \% \mathrm{CO}_{2}$. RASMCs at passage 3-6 were used for subsequent experiments. Following transfection, cells were treated with recombinant human VEGF protein $(10 \mathrm{ng} / \mathrm{ml}$; R\&D Systems China Co., Ltd.) for 5,15 or $30 \mathrm{~min}$ at $37^{\circ} \mathrm{C}$.

Sample collection. In the present study, all volunteers were recruited from Fuwai Hospital Chinese Academy of Medical Sciences Shenzhen, although volunteers were from residence across China. Pathological aortic tissues from 20 patients with AD (age, 45-62 years; 16 male patients and 4 female patients) and healthy aortic tissues from 5 patients who underwent coronary artery bypass grafting surgery (age, 55-74 years; 3 male patients and 2 female patients) were collected between October 2018 and October 2019. The following inclusion criteria were used in the present study: Pain symptoms for $<48 \mathrm{~h}$; and diagnosed with acute AD after aorta computed tomography angiography examination. The following exclusion criteria were used in the present study: Heart failure, acute myocardial infarction, connective tissue disease or tumor disease.
The present study was approved by the Research Ethics Committee of Fuwai Hospital Chinese Academy of Medical Sciences Shenzhen (approval no. SP2019004). Written informed consent was obtained from each patient.

Reverse transcription-quantitative PCR (RT-qPCR). Total RNA was extracted from each sample using TRIzol ${ }^{\circledR}$ (Invitrogen; Thermo Fisher Scientific, Inc.) according to the manufacturer's protocol. Total RNA was reverse transcribed into cDNA using PrimeScript ${ }^{\mathrm{TM}}$ RT Master Mix [cat.no.RR036A; Takara Biomedical Technology (Beijing) Co., Ltd.] according to the manufacturer's protocol. Subsequently, qPCR was performed using SYBR green fluorescence [Takara Biomedical Technology (Beijing) Co., Ltd.]. The following primers were used for qPCR: OGN forward, 5'-TCTACACTT CTCCTGTTACTGCT-3' and reverse, 5'-GAGGTGGTGGTG TT-ATTGCCTCA-3'; and GAPDH forward, 5'-GGCAGTGAT GGCATGGACTGT-3' and reverse, 5'-CCTTCATTGACC TCAACTACA-3'. The following thermocycling conditions were used for qPCR: $95^{\circ} \mathrm{C}$ for $2 \mathrm{~min}$; followed by 40 cycles of $95^{\circ} \mathrm{C}$ for $5 \mathrm{sec}, 60^{\circ} \mathrm{C}$ for $30 \mathrm{sec}$ and $72^{\circ} \mathrm{C}$ for $60 \mathrm{sec}$. mRNA expression levels were quantified using the $2^{-\Delta \Delta \mathrm{Cq}}$ method (26) and normalized to the internal reference gene GAPDH.

Western blotting. Total protein was extracted from tissues or cultured cells using RIPA lysis buffer (Beijing Solarbio Science \& Technology Co., Ltd.). Protein concentration was determined using a BCA Protein Assay kit (Beijing Solarbio Science \& Technology Co., Ltd.). Equal amounts of protein $(30 \mu \mathrm{g})$ were separated via 10\% SDS-PAGE and transferred to PVDF membranes. Following blocking with 5\% skimmed milk in TBST $(0.05 \%$ Tween-20) for $1 \mathrm{~h}$ at room temperature, the membranes were incubated at $4^{\circ} \mathrm{C}$ overnight with the following primary antibodies: anti-GAPDH (cat. no. 5174; 1:10,000; Cell Signaling Technology, Inc.), anti-Tubulin (cat. no. 2148; 1:10,000; Cell Signaling Technology, Inc.), anti-OGN (cat. no. ab211456; 1:2,000; Abcam), anti-phosphorylated (p)-AKT (cat. no. 4060; 1:2,000; Cell Signaling Technology, Inc.), anti-total AKT (cat. no. 9272; 1:2,000; Cell Signaling Technology, Inc.), anti-p-ERK1/2 (cat. no. 9102; 1:1,000; Cell Signaling Technology, Inc.), anti-total ERK1/2 (cat. no. 9101; 1:1,000; Cell Signaling Technology, Inc.), anti-p-VEGFR2 (cat. no. 2478; 1:2,000; Cell Signaling Technology, Inc.) and anti-total VEGFR2 (cat. no. 9698; 1:2,000; Cell Signaling Technology, Inc.). After washing with TBST for three times for $5 \mathrm{~min}$ each, the membranes were incubated with an anti-rabbit IgG (cat. no. 7074; 1:10,000; Cell Signaling Technology, Inc.) secondary antibody for $1 \mathrm{~h}$ at room temperature. Following washing with TBST for three times for 5 min each, protein bands were visualized using SuperSignal West Femto Maximum Sensitivity Substrate (Thermo Fisher Scientific, Inc.) and an iBright bioimaging system (Invitrogen; Thermo Fisher Scientific, Inc.). Protein expression levels were semi-quantified using Quantity One Analysis software (version 4.0; Bio-Rad Laboratories, Inc.) with GAPDH or Tubulin as the loading control.

Transfection of OGN-targeting small interfering (si) $R N A$. At $80 \%$ confluence, RASMCs were transfected with $50 \mathrm{nM}$ OGN-specific siRNA or control siRNA using 

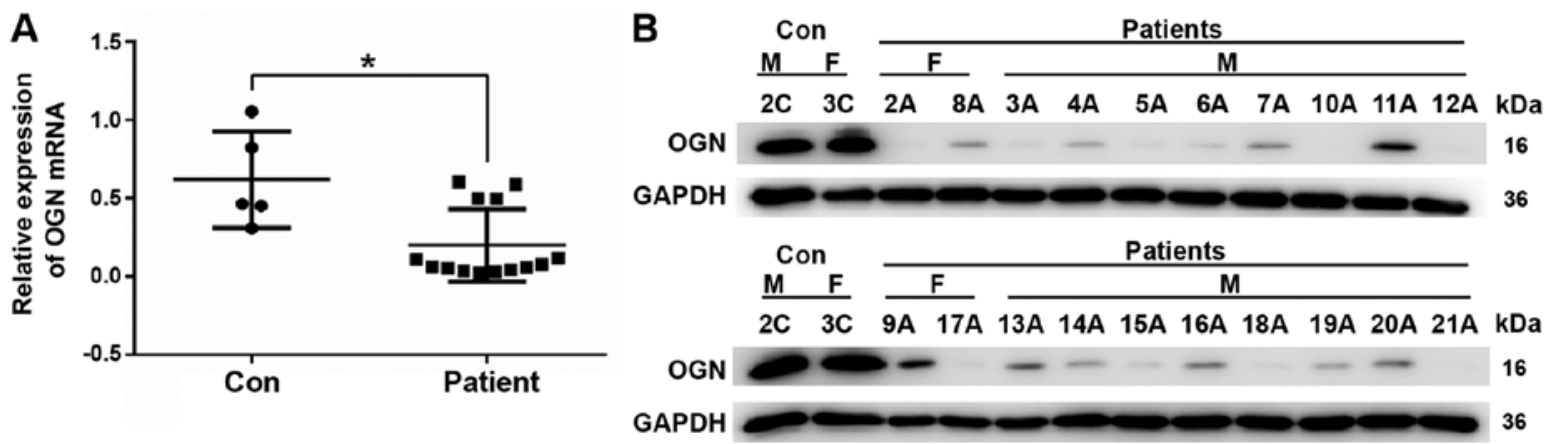

Figure 1. OGN expression levels in patients with AD and healthy control subjects. Thoracic aortic tissues from 20 patients with AD and healthy thoracic aortic tissues from 5 control subjects were collected. In two of the healthy thoracic aortic tissue samples, total protein extraction and OGN protein expression analysis failed. OGN (A) mRNA and (B) protein expression levels. * P<0.05. OGN, osteoglycin; AD, aortic dissection; Con, control; M, male; F, female; C, control; A, AD.

Lipofectamine ${ }^{\circledR} 2000$ (Invitrogen; Thermo Fisher Scientific, Inc.) at $37^{\circ} \mathrm{C}$ for $48 \mathrm{~h}$. The OGN-specific siRNA (si-OGN; forward, 5'-GUGCCCACCAAGAAAGAAATT-3' and reverse, 5'-UUUCUUUCUUGGUGGGCACTT-3') and control siRNA [si-negative control (NC); non-specific; forward, 5'-ACAACG AACAAGCGAACAACA-3' and reverse, 5'-UGUUGUUCG CUUGUUCGUUGU-3'] were synthesized by Sangon Biotech Co., Ltd. Cells were divided into three groups: i) Blank, cells were left untreated; ii) NC, cells were transfected with si-NC; and iii) OGN (marked as OGN-Rat-401), cells were transfected with si-OGN. At $48 \mathrm{~h}$ post-transfection, cells were used for subsequent experiments.

Cell proliferation assay. The effect of OGN knockdown on RASMC viability was assessed by performing a Cell Counting Kit-8 (CCK-8) assay. Briefly, RASMCs were seeded $\left(2.5 \times 10^{3}\right.$ cells/well) into 96 -well plates. At $48 \mathrm{~h}$ post-transfection, platelet-derived growth factor-BB (PDGF-BB; final concentration, $60 \mu \mathrm{g} / \mathrm{l}$; Cyagen Biosciences, Inc.) was added to the culture medium to stimulate cell proliferation for $24 \mathrm{~h}$ at $37^{\circ} \mathrm{C}$. Subsequently, $10 \mu \mathrm{l} \mathrm{CCK}-8$ reagent (Dojindo Molecular Technologies, Inc.) was added to each well and incubated in the dark for $1.5 \mathrm{~h}$ at $37^{\circ} \mathrm{C}$. The optical density was measured at a wavelength of $450 \mathrm{~nm}$ using a microplate reader (Promega Corporation).

A BrdU immunofluorescence assay was performed on OGN-knockdown RASMCs. RASMCs were seeded ( $2 \times 10^{4}$ cells/well) into 6 -well plates. At $48 \mathrm{~h}$ post-transfection, cells were incubated with $50 \mu \mathrm{M} \mathrm{BrdU}$ for $12 \mathrm{~h}$ at $37^{\circ} \mathrm{C}$ in humidified incubator with $5 \% \mathrm{CO}_{2}$. Cells were fixed with $4 \%$ paraformaldehyde at room temperature for $20 \mathrm{~min}$. Subsequently, cells were washed with PBS and desaturated with $2 \mathrm{M} \mathrm{HCl}$ at $37^{\circ} \mathrm{C}$ for $10 \mathrm{~min} . \mathrm{H}_{3} \mathrm{BO}_{3}(\mathrm{pH} 8.5)$ was used for renaturation at room temperature for $10 \mathrm{~min}$. Samples were washed three times with PBS for $5 \mathrm{~min}$. Cells were permeabilised using $0.5 \%$ Triton $\mathrm{X}-100$ at room temperature for $15 \mathrm{~min}$. Cells were washed three times with PBS for $5 \mathrm{~min}$, blocked with non-immunized goat serum (Beijing Dingguo Changsheng Biotechnology Co., Ltd.; 1:1,000) at $37^{\circ} \mathrm{C}$ for $30 \mathrm{~min}$ and incubated with a BrdU primary antibody (cat. no. ab8152; 1:100; Abcam) overnight at $4^{\circ} \mathrm{C}$. Following washing three times with PBS for $5 \mathrm{~min}$, cells were incubated with the secondary antibody (cat. no. 8890; 1:1,000; Cell Signaling Technology, Inc.) at $37^{\circ} \mathrm{C}$ for $2 \mathrm{~h}$. Samples were washed three times with PBS and then were incubated with DAPI (1:100) at room temperature for $5 \mathrm{~min}$. Following two washes with PBS for $5 \mathrm{~min}$, samples were washed with distilled water. The slides were dried and sealed with $50 \%$ glycerin. Stained sections were observed in five fields of view using an LH-M100CB inverted fluorescence microscope (Nikon Corporation; magnification, x200).

Wound healing assay. For the wound healing assay, RASMCs were seeded into 6-well plates. At $95 \%$ confluence, the cell monolayer was mechanically scraped using a sterile pipette tip to create a single scratch. Cells were maintained in serum-free medium. At 0, 24 and $48 \mathrm{~h}$, the wound was observed using a light optical microscope (magnification, $x 40$ ). The results are presented as the distance of wound healing, which was measured using ImageJ software (version 1.8.0.112; National Institutes of Health).

Cell invasion assay. RASMCs were seeded ( $5 \times 10^{4}$ cells/well) into the upper chamber of the Matrigel-coated Transwell plate (pore size, $8 \mu \mathrm{m}$ ). DMEM supplemented with 10\% FBS was plated into the lower chambers. Following incubation for $48 \mathrm{~h}$ at $37^{\circ} \mathrm{C}$, invading cells were fixed with $4 \%$ paraformaldehyde at room temperature for $20 \mathrm{~min}$, and stained with $0.5 \%$ crystal violet solution at room temperature for $10 \mathrm{~min}$. Invading cells were visualized in five randomly selected fields of view using a light microscope (magnification, x200).

Statistical analysis. Statistical analyses were performed using GraphPad Prism software (version 6; GraphPad Software, Inc.). Comparisons among multiple groups were analyzed using one-way ANOVA followed by Dunnett's post hoc test. Comparisons between two groups were analyzed using an unpaired Student's t-test. Data are presented as the mean \pm SD of three independently repeated experiments. $\mathrm{P}<0.05$ was considered to indicate a statistically significant difference.

\section{Results}

OGN is downregulated in aortic tissues from patients with $A D$. OGN mRNA expression levels were significantly lower in the thoracic aortic tissues of 20 patients with AD compared with the healthy thoracic aortic tissues of 5 control subjects (Fig. 1A). The western blotting results demonstrated that the protein expression levels of OGN in the thoracic aortic tissues 


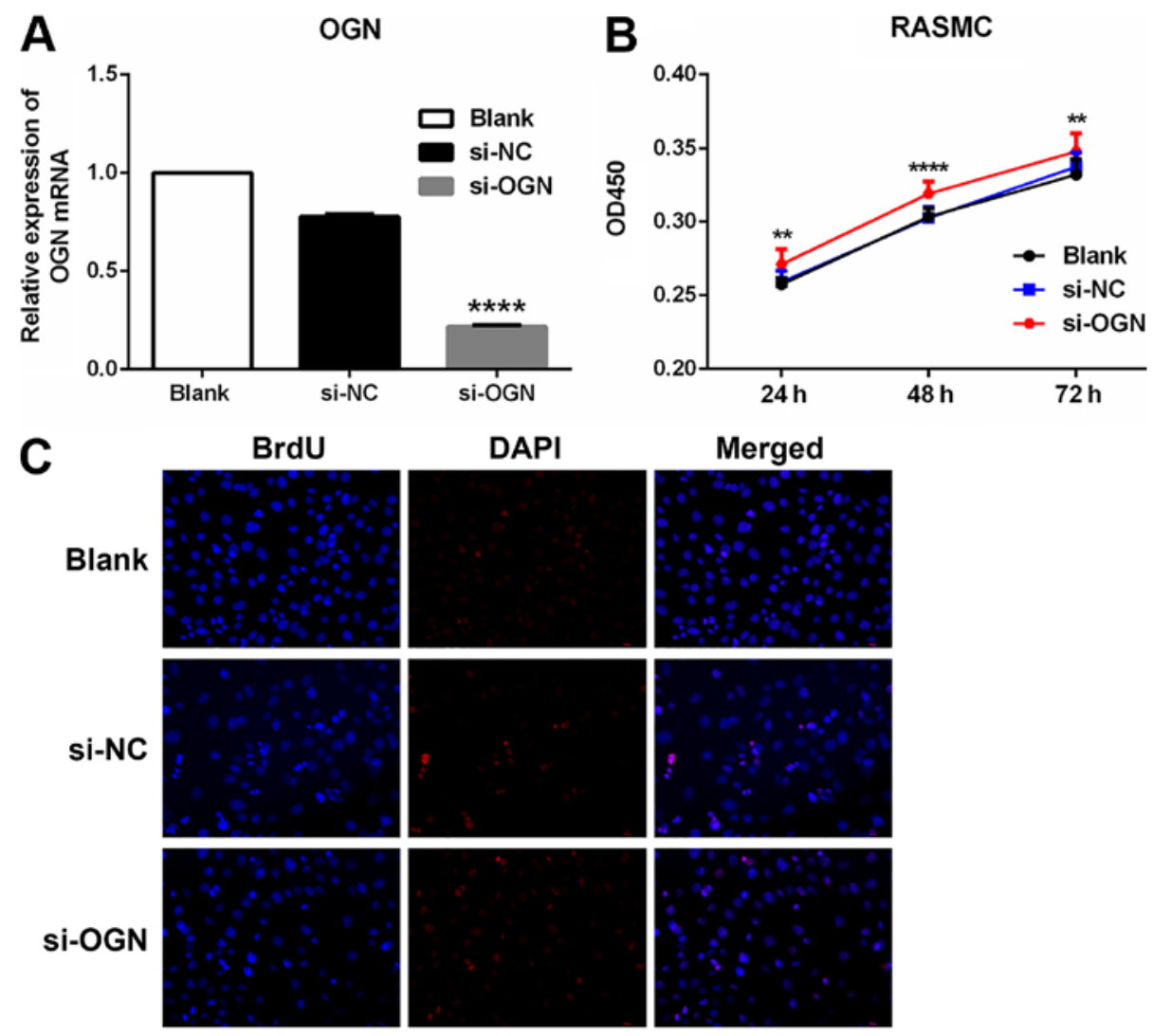

Figure 2. Effect of OGN knockdown on RASMC proliferation. Cells were divided into three groups: i) Blank, cells were left untreated; ii) NC, cells were transfected with si-NC; and iii) OGN (marked as OGN-Rat-401), cells were transfected with si-OGN. Transfected RASMCs were seeded into 96-well plates and stimulated with $60 \mu \mathrm{g} / 1$ platelet-derived growth factor-BB. (A) Transfection efficiency of si-OGN. (B) Cell proliferation was determined by performing the Cell Counting Kit-8 assay. (C) Cell proliferation was also assessed by performing BrdU immunofluorescence staining (magnification, $\mathrm{x} 200)$. ${ }^{* *} \mathrm{P}<0.01$ and ${ }^{* * * * *} \mathrm{P}<0.0001$ vs. si-NC. OGN, osteoglycin; RASMC, rat aortic smooth muscle cell; NC, negative control; si, small interfering RNA; OD, optical density.

A

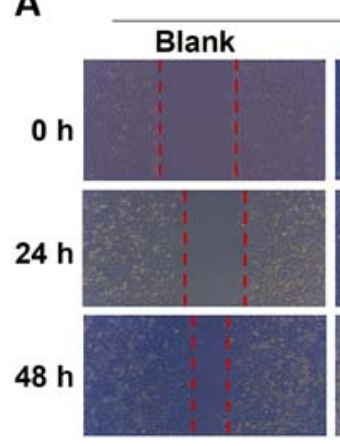

B

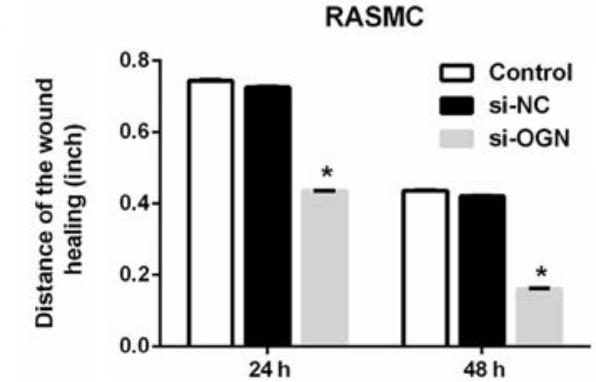

Figure 3. Effect of OGN knockdown on RASMC migration. si-OGN-transfected RASMCs were seeded into 6-well plates to form confluent monolayers. A sterile pipette was used to form a single scratch in the cell monolayer. The wound was observed at 0,24 and $48 \mathrm{~h}$ using an optical microscope. Cell migration was (A) determined by performing a wound healing assay (magnification, $\mathrm{x} 40$ ) and (B) quantified. ${ }^{*} \mathrm{P}<0.05$ vs. si-NC. OGN, osteoglycin; RASMC, rat aortic smooth muscle cell; si, small interfering RNA; NC, negative control. from 20 patients with $\mathrm{AD}$ were markedly reduced compared with the thoracic aortic tissues from 3 out of the 5 control subjects (Fig. 1B).

OGN knockdown enhances RASMC proliferation and migration. Following transfection with si-OGN, OGN mRNA expression levels were significantly decreased compared with the si-NC group (Fig. 2A). The CCK-8 assay was performed to investigate whether OGN knockdown affected PDGF-BB-induced cell proliferation and survival. OGN knockdown significantly enhanced RASMC proliferation at 24, 48 and $72 \mathrm{~h}$ compared with the si-NC group (Fig. 2B). The immunofluorescence assay displayed similar results (Fig. 2C); cell proliferation was also notably increased in the si-OGN group compared with the si-NC group.

To assess the effect of OGN on RASMC migration, wound healing and Transwell invasion assays were performed. Compared with the si-NC group, OGN knockdown significantly decreased the width of the wound by 41.1 and $61.9 \%$ at 24 and $48 \mathrm{~h}$, respectively (Fig. 3A and B). Moreover, the number of invading RASMCs was decreased by $\sim 30 \%$ in the si-OGN group compared with the si-NC group (Fig. 4A and B). The results indicated that OGN knockdown was associated with increased RASMC proliferation and migration, and decreased invasion.

OGN knockdown further increases the phosphorylation of $A K T$ and ERK1/2 in VEGF-treated RASMCs. In mouse models 
A

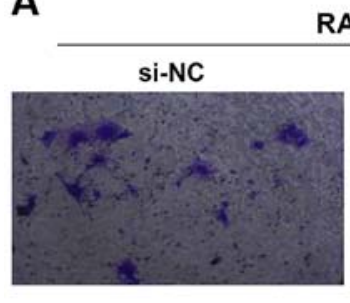

RASMC

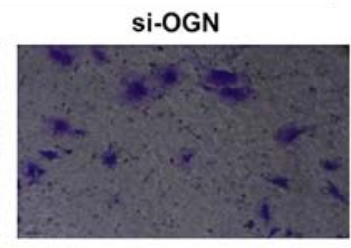

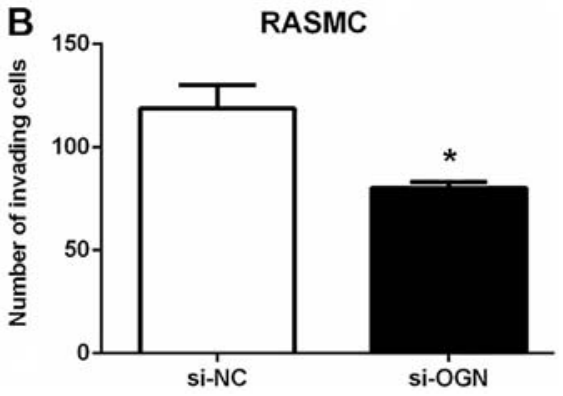

Figure 4. Effect of OGN knockdown on RASMC invasion. si-OGN transfected RASMCs were seeded (5x10 4 cells/well) into the upper chamber of the Transwell plate. DMEM supplemented with $10 \%$ FBS was plated into the lower chambers. Following incubation for 48 h, invading cells were fixed with cold methanol and $4 \%$ paraformaldehyde, and stained with $0.5 \%$ crystal violet solution. Invading cells were observed in five randomly selected fields of view. Cell invasion was (A) determined by performing a Transwell invasion assay (magnification, $\mathrm{x} 200$ ) and (B) quantified. * $\mathrm{P}<0.05$ vs. si-NC. OGN, osteoglycin; RASMC, rat aortic smooth muscle cell; si, small interfering RNA; NC, negative control.
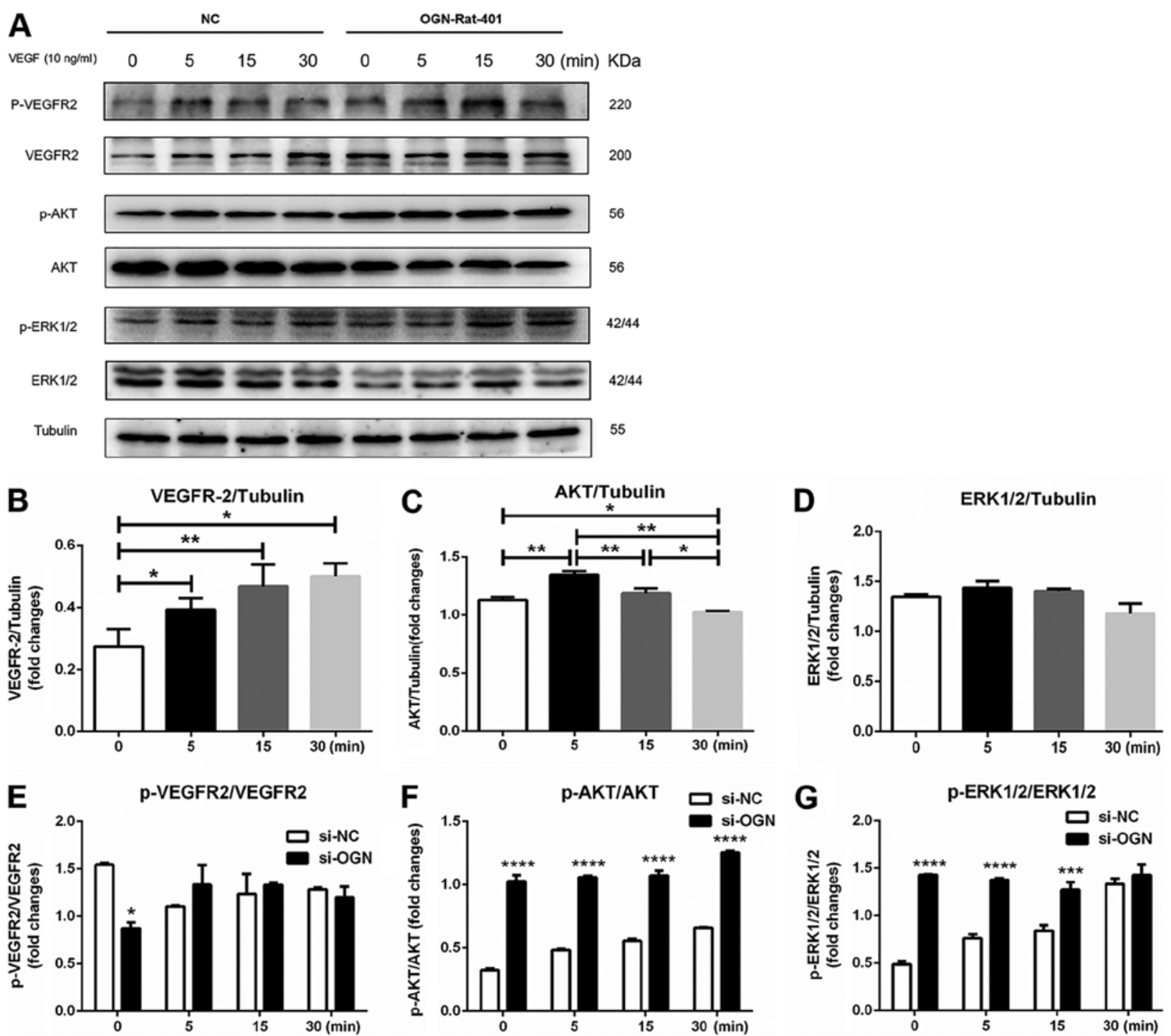

Figure 5. OGN regulates the VEGFR/AKT signaling pathway. Cells were transfected with si-OGN or si-NC for $48 \mathrm{~h}$. Subsequently, cells were treated with VEGF (10 ng/ml) for 5, 15 or $30 \mathrm{~min}$. Protein expression levels were (A) determined via western blotting and semi-quantified for (B) VEGFR2, (C) AKT, (D) ERK1/2, (in si-NC-transfected cells) (E) p-VEGFR2/VEGFR2, (F) p-AKT/AKT and (G) p-ERK1/2/ERK1/2. ${ }^{*} \mathrm{P}<0.05,{ }^{* *} \mathrm{P}<0.01,{ }^{* * *} \mathrm{P}<0.001$ and ${ }^{* * * *} \mathrm{P}<0.0001$ vs. si-NC. OGN, osteoglycin; si, small interfering RNA; NC, negative control; p, phosphorylated.

of limb ischemia, increased expression levels of OGN are closely related to inhibition of the VEGF/VEGFR2 signaling pathway $(27,28)$. In the present study, OGN-knockdown
RASMCs displayed significantly reduced VEGFR2 phosphorylation compared with si-NC-transfected RASMCs (Fig. 5A and E). VEGF-mediated stimulation of 
si-NC-transfected RASMCs significantly increased the levels of VEGPR2, and increased the expression level of AKT at 5 min compared with $0 \mathrm{~min}$, which returned to the baseline level at $30 \mathrm{~min}$ (Fig. 5A, B and C). The protein expression levels of ERK1/2 were not significantly altered among the different groups (Fig. 5A and D). In si-NC-transfected RASMCs, VEGF stimulation notably increased the phosphorylation levels of ERK1/2 and AKT in a time-dependent manner (Fig. 5A, F and G). Moreover, ERK1/2 and AKT phosphorylation levels were significantly increased in si-OGN-transfected RASMCs compared with si-NC-transfected RASMCs, although a significant difference in ERK1/2 phosphorylation between the si-NC and si-OGN groups was not observed following $30 \mathrm{~min}$ VEGF stimulation (Fig. 5A, F and G). The results suggested that OGN knockdown facilitated VEGF-induced activation of AKT and ERK1/2.

\section{Discussion}

In the present study, the results demonstrated that OGN expression levels were significantly downregulated in patients with AD compared with healthy controls. Furthermore, the results suggested that OGN expression was negatively associated with cell proliferation and migration. Inspired by our previous work and other previous studies, the present study indicated that OGN may regulate the downstream signaling molecules AKT and ERK1/2 via the VEGF/VEGFR2 signaling pathway, thereby affecting cell proliferation, migration and angiogenesis.

Increasing evidence has demonstrated that the altered expression of proteoglycans is associated with degeneration of the aortic wall in AD (29). Versican, a large chondroitin sulfate proteoglycan, is required for RASMC proliferation and migration, and its degradation leads to fragmentation of elastin and predisposition to $\mathrm{AD}(30,31)$. Genetic depletion of biglycan, a member of the class I family of SLRPs, results in spontaneous AD and rupture (32). Alterations in the expression levels of OGN, which was originally identified as a modulator of bone formation, have been implicated in atherosclerosis, myocardial fibrosis, schaemia-induced angiogenesis and other vascular diseases $(28,33,34)$. The expression levels of OGN in aortas are increased in adult rats at 2 weeks post-balloon injury and in 2-week-old neonatal rats (33). OGN mRNA expression levels are downregulated in rat RASMCs stimulated with basic fibroblast growth factor, TGF $\beta$, PDGF and angiotensin II (33). Similarly, OGN expression levels are decreased in the hypertrophic aortas of sinoaortic-denervated rats (35). Consistent with the aforementioned studies, the present study demonstrated that OGN expression levels were significantly downregulated in the thoracic aortic tissues of patients with AD compared with healthy controls, suggesting that OGN might be involved in the pathological progression of AD.

Aortic vascular smooth muscle cells, the predominant cell type in the medial layer of the aortic wall, serve an important role in maintaining structural integrity and regulating vascular tone (36). RASMC proliferation and migration occur in response to various vascular injuries, and contribute to the development of pathological remodeling and vascular diseases (37). Mutations in genes encoding proteins required for SMC contraction leads to the occurrence of thoracic aortic aneurysms and dissections (38). The switch from a contractile
RASMC phenotype to a synthetic, migratory and proliferative RASMC phenotype is a pivotal contributor to the development of AD (39). The present study indicated that compared with si-NC-transfected RASMCs, OGN-knockdown RASMCs displayed enhanced proliferation and migration, which may facilitate the development of AD. Previous studies have indicated that OGN is closely related to proliferation and migration in a number of different cell types $(40,41)$. OGN overexpression reduces proliferation and inhibits invasion in human colon cancer cell lines $(20,42)$. In the present study, compared with si-NC, OGN knockdown increased RASMC migration but decreased invasion. Therefore, it was hypothesized that RASMCs might respond to multiple growth factors, inflammatory cytokines and vasoactive substances, leading to OGN downregulation, which promotes cell proliferation and migration, and thereby modulating AD progression. The different effects of OGN knockdown on the invasion abilities of RASMCs and colon cancer cells may be attributed to the distinct extracellular stimuli and intracellular signaling pathways of the two cell types.

The VEGF/VEGFR axis is involved in numerous physiological and pathological processes, including embryologic development, normal growth, tissue repair and tumorigenesis $(43,44)$. In the process of angiogenesis, VEGF binds to VEGFR on endothelial cells, resulting in endothelial cell proliferation and migration (45). OGN competitively binds to VEGFR2 in cultured human umbilical vein endothelial cells (HUVECs) (46), and a coimmunoprecipitation assay confirmed the direct interaction between OGN and VEGFR2. Wu et al (28) further reported that OGN negatively modulates the activation of VEGFR2 and its downstream signaling pathways. In the present study, no significant alterations in the levels of phosphorylated VEGFR2 after exposure to VEGF for 5-30 min were observed between si-NC- and si-OGN-transfected RASMCs; however, VEGF-induced activation of AKT and ERK1/2 was significantly enhanced in OGN-knockdown cells compared with si-NC-transfected cells. AKT and ERK1/2 are important downstream signaling molecules that are required for the VEGFR2-induced proliferation and migration of lymphatic endothelial cells and human brain RASMCs $(47,48)$. The results of the present study indicated that compared with the si-NC group, OGN knockdown significantly enhanced the phosphorylation of AKT and ERK1/2 in RASMCs, which triggered cell proliferation and migration. The results of the present study were consistent with the finding that OGN knockdown in HUVECs resulted in enhanced AKT and ERK1/2 phosphorylation in response to VEGF (46).

Other VSMC-related mechanisms may exist in AD. ECM softening serves a pivotal role in regulating the VSMC phenotype switch and provides a potential target for treating VSMC dysfunction and AD disease, which has been reported in Cardiovascular Toxicology (49). The aforementioned study focused on synthetic phenotype-related genes and ECM softening phenotype, whereas the present study focused on the role of OGN in VSMC proliferation and migration, providing two possible mechanisms underlying AD. The two identified mechanisms may display cross-talk and share certain targets and factors, but further investigation is required. Synthetic phenotype-related genes, including osteopontin, matrix 
metalloproteinases and inflammatory cytokines, are upregulated in VSMCs (49). On the other hand, OGN is a component of the vascular extracellular matrix and may also influence the vascular system (50). Therefore, it was hypothesized that OGN may contribute to ECM softening in the regulation of the VSMC phenotype switch.

The present study had a number of limitations. First, the number of samples included in the present study was limited due to the low incidence of AD. The RASMC model used in the present study is a common tool used in AD research (25), but cell proliferation and migration can occur in a number of other vascular diseases, such as atherosclerosis and vascular neointima formation $(51,52)$. In addition, the function of OGN in $\mathrm{AD}$ may be related to the process of angiogenesis. However, no angiogenesis-related genes were analyzed in the AD tissues or in the RASMC model in the present study. If angiogenesis is the target of OGN, further research in vascular endothelial cells rather than vascular smooth muscle cells is required. Therefore, future studies should investigate the effect of OGN on angiogenesis in $\mathrm{AD}$ to reveal the possible underlying mechanisms.

In conclusion, the present study suggested that OGN knockdown facilitated the stimulatory effect of PDGF-BB on RASMC proliferation and migration. The results indicated that OGN regulated the VEGF/VEGFR2 axis and the downstream signaling molecules AKT and ERK1/2, thus affecting the biological activity of RASMCs. Therefore, OGN may serve as a novel therapeutic target for AD.

\section{Acknowledgements}

Not applicable.

\section{Funding}

The present study was supported by the National Natural Science Foundation of China (grant nos. 81600208, 81970210 and 81570256), the Medical Scientific Research Foundation of Guangdong Province of China (grant no. A2018019) and the Science and Technology Project of Shenzhen of China (grant nos. JCYJ20180302173909492, JCYJ20180508152222104 and KQJSCX20180329104902378).

\section{Availability of data and materials}

The datasets used and/or analyzed during the current study are available from the corresponding author on reasonable request.

\section{Authors' contributions}

ZW and MW performed the literature review, wrote the initial draft and analyzed the data. XZ and BC performed the experiments and collected the data. MW provided ideas and recommendations, designed the research and reviewed the manuscript. All authors read and approved the final manuscript.

\section{Ethics approval and consent to participate}

All the procedures involving human participants were performed in accordance with the ethical standards of the institutional and the 1964 Declaration of Helsinki and its later amendments or comparable ethical standards. The present study was approved by the Ethics Committee of Fuwai Hospital Chinese Academy of Medical Sciences Shenzhen (approval no. SP2019004). All patients signed an informed consent form, which was approved by the Institutional Review Board.

\section{Patient consent for publication}

Not applicable.

\section{Competing interests}

The authors declare that they have no competing interests.

\section{References}

1. Clough RE and Nienaber CA: Management of acute aortic syndrome. Nat Rev Cardiol 12: 103-114, 2015.

2. Sherrah AG, Grieve SM, Jeremy RW, Bannon PG, Vallely MP and Puranik R: MRI in chronic aortic dissection: A systematic review and future directions. Front Cardiovasc Med 2: 5, 2015.

3. Divchev D, Najjar T, Tillwich F, Aboukoura M, Rehders T and Nienaber CA: Risk assessment of acute aortic dissection. Dtsch Med Wochenschr 139: 1947-1951, 2014 (In German).

4. Ridge CA and Litmanovich DE: Acute aortic syndromes: Current status. J Thorac Imaging 30: 193-201, 2015.

5. Segreto A, Chiusaroli A, De Salvatore S and Bizzarri F: Biomarkers for the diagnosis of aortic dissection. J Card Surg 29: 507-511, 2014.

6. Kumar A and Allain RM: Aortic dissection. Critical Care Secrets (Fifth Edition): 204-211, 2013.

7. Elsayed RS, Cohen RG, Fleischman F and Bowdish ME: Acute type A aortic dissection. Cardiol Clin 35: 331-345, 2017.

8. Gawinecka J, Schönrath F and von Eckardstein A: Acute aortic dissection: Pathogenesis, risk factors and diagnosis. Swiss Med Wkly 147: w14489, 2017.

9. Evangelista A, Isselbacher EM, Bossone E, Gleason TG, Eusanio MD, Sechtem U, Ehrlich MP, Trimarchi S, Braverman AC, Myrmel T, et al: Insights from the international registry of acute aortic dissection: A 20-Year experience of collaborative clinical research. Circulation 137: 1846-1860, 2018.

10. Wen D, Zhou XL, Li JJ and Hui RT: Biomarkers in aortic dissection. Clin Chim Acta 412: 688-695, 2011.

11. Jiang WJ, Ren WH, Liu XJ, Liu Y, Wu FJ, Sun LZ, Lan F, Du J and Zhang HJ: Disruption of mechanical stress in extracellular matrix is related to Stanford type A aortic dissection through down-regulation of Yes-associated protein. Aging (Albany NY) 8: 1923-1939, 2016.

12. Gentili C and Cancedda R: Cartilage and bone extracellular matrix. Curr Pharm Des 15: 1334-1348, 2009.

13. Wu D, Shen YH, Russell L, Coselli JS and LeMaire SA: Molecular mechanisms of thoracic aortic dissection. J Surg Res 184: 907-924, 2013.

14. Billaud M, Hill JC, Richards TD, Gleason TG and Phillippi JA: Medial hypoxia and adventitial vasa vasorum remodeling in human ascending aortic aneurysm. Front Cardiovasc Med 5: 124, 2018.

15. Wang Z, Zhuang X, Chen B, Wen J, Peng F, Liu X and Wei M: 99-case study of sporadic aortic dissection by whole exome sequencing indicated novel disease-associated genes and variants in Chinese population. Biomed Res Int 2020: 7857043, 2020.

16. Zhu L, Vranckx R, Khau Van Kien P, Lalande A, Boisset N, Mathieu F, Wegman M, Glancy L, Gasc JM, Brunotte F, et al: Mutations in myosin heavy chain 11 cause a syndrome associating thoracic aortic aneurysm/aortic dissection and patent ductus arteriosus. Nat Genet 38: 343-349, 2006.

17. Marshall LM, Carlson EJ, O'Malley J, Snyder CK, Charbonneau NL, Hayflick SJ, Coselli JS, Lemaire SA and Sakai LY: Thoracic aortic aneurysm frequency and dissection are associated with fibrillin-1 fragment concentrations in circulation. Circ Res 113: 1159-1168, 2013.

18. Seike Y, Minatoya K, Sasaki H, Tanaka H, Itonaga T, Inoue Y, Morisaki H, Morisaki T, Ishibashi-Ueda H and Kobayashi J: Clinical outcomes of aortic repair in young adult patients with ACTA2 mutations. Gen Thorac Cardiovasc Surg 65: 686-691, 2017. 
19. Costa RA, Martins RST, Capilla E, Anjos L and Power DM: Vertebrate SLRP family evolution and the subfunctionalization of osteoglycin gene duplicates in teleost fish. BMC Evol Biol 18: $191,2018$.

20. Hu X, Li YQ, Li QG, Ma YL, Peng JJ and Cai SJ: Osteoglycin-induced VEGF inhibition enhances T lymphocytes infiltrating in colorectal cancer. EBioMedicine 34: 35-45, 2018.

21. Ge G, Seo NS, Liang X, Hopkins DR, Höök M and Greenspan DS: Bone morphogenetic protein-1/tolloid-related metalloproteinases process osteoglycin and enhance its ability to regulate collagen fibrillogenesis. J Biol Chem 279: 41626-41633, 2004.

22. Deckx S, Heymans S and Papageorgiou AP: The diverse functions of osteoglycin: A deceitful dwarf, or a master regulator of disease? FASEB J 30: 2651-2661, 2016.

23. Seki T, Saita E, Kishimoto Y, Ibe S, Miyazaki Y, Miura K, Ohmori R, Ikegami Y, Kondo K and Momiyama Y: Low levels of plasma osteoglycin in patients with complex coronary lesions J Atheroscler Thromb 25: 1149-1155, 2018.

24. Matsumoto K, Maniwa T, Tanaka T, Satoh K, Okunishi H and Oda T: Proteomic analysis of calcified abdominal and thoracic aortic aneurysms. Int J Mol Med 30: 417-429, 2012.

25. Wang Z, Zhuang X, Chen B, Feng D, Li G and Wei M: The role of miR-107 as a potential biomarker and cellular factor for acute aortic dissection. DNA Cell Biol 39: 1895-1906, 2020.

26. Livak KJ and Schmittgen TD: Analysis of relative gene expression data using real-time quantitative PCR and the 2(-Delta Delta C(T)) method. Methods 25: 402-408, 2001

27. Hu X, Li YQ, Li QG, Ma YL, Peng JJ and Cai SJ: Osteoglycin (OGN) reverses epithelial to mesenchymal transition and invasiveness in colorectal cancer via EGFR/Akt pathway. J Exp Clin Cancer Res 37: 41, 2018.

28. Wu QH, Ma Y, Ruan CC, Yang Y, Liu XH, Ge Q, Kong LR, Zhang JW, Yan C and Gao PJ: Loss of osteoglycin promotes angiogenesis in limb ischaemia mouse models via modulation of vascular endothelial growth factor and vascular endothelial growth factor receptor 2 signalling pathway. Cardiovasc Res 113: 70-80, 2017.

29. Cikach FS, Koch CD, Mead TJ, Galatioto J, Willard BB, Emerton KB, Eagleton MJ, Blackstone EH, Ramirez F, Roselli EE and Apte SS: Massive aggrecan and versican accumulation in thoracic aortic aneurysm and dissection. JCI Insight 3: e97167, 2018.

30. Evanko SP, Angello JC and Wight TN: Formation of hyaluronanand versican-rich pericellular matrix is required for proliferation and migration of vascular smooth muscle cells. Arterioscler Thromb Vasc Biol 19: 1004-1013, 1999.

31. Wight TN: Arterial remodeling in vascular disease: A key role for hyaluronan and versican. Front Biosci 13: 4933-4937, 2008

32. Heegaard AM, Corsi A, Danielsen CC, Nielsen KL, Jorgensen HL, Riminucci M, Young MF and Bianco P: Biglycan deficiency causes spontaneous aortic dissection and rupture in mice. Circulation 115: 2731-2738, 2007.

33. Shanahan CM, Cary NR, Osbourn JK and Weissberg PL: Identification of osteoglycin as a component of the vascular matrix. Differential expression by vascular smooth muscle cells during neointima formation and in atherosclerotic plaques. Arterioscler Thromb Vasc Biol 17: 2437-2447, 1997.

34. Deckx S, Heggermont W, Carai P, Rienks M, Dresselaers T, Himmelreich U, van Leeuwen R, Lommen W, van der Velden J, Gonzalez A, et al: Osteoglycin prevents the development of age-related diastolic dysfunction during pressure overload by reducing cardiac fibrosis and inflammation. Matrix Biol 66: $110-124,2018$

35. Gu XS, Lei JP, Shi JB, Lian WL, Yang X, Zheng X and Qin YW: Mimecan is involved in aortic hypertrophy induced by sinoaortic denervation in rats. Mol Cell Biochem 352: 309-316, 2011.
36. Korneva A, Humphrey JD: Maladaptive aortic remodeling in hypertension associates with dysfunctional smooth muscle contractility. Am J Physiol Heart Circ Physiol 316: H265-H278, 2019.

37. Wang D, Wang Q, Yan G, Qiao Y and Tang C: Phloretin inhibits platelet-derived growth factor-BB-induced rat aortic smooth muscle cell proliferation, migration, and neointimal formation after carotid injury. J Cardiovasc Pharmacol 65: 444-455, 2015.

38. Milewicz DM, Trybus KM, Guo DC, Sweeney HL, Regalado E, Kamm K and Stull JT: Altered smooth muscle cell force generation as a driver of thoracic aortic aneurysms and dissections. Arterioscler Thromb Vasc Biol 37: 26-34, 2017.

39. Inamoto S, Kwartler CS, Lafont AL, Liang YY, Fadulu VT, Duraisamy S, Willing M, Estrera A, Safi H, Hannibal MC, et al: TGFBR2 mutations alter smooth muscle cell phenotype and predispose to thoracic aortic aneurysms and dissections. Cardiovasc Res 88: 520-529, 2010.

40. Zuo C, Li X, Huang J, Chen D, Ji K, Yang Y, Xu T, Zhu D, Yan $\mathrm{C}$ and Gao P: Osteoglycin attenuates cardiac fibrosis by suppressing cardiac myofibroblast proliferation and migration through antagonizing lysophosphatidic acid 3/matrix metalloproteinase 2/epidermal growth factor receptor signalling. Cardiovasc Res 114: 703-712, 2018.

41. Xu T, Zhang R, Dong M, Zhang Z, Li H, Zhan C and Li X: Osteoglycin (OGN) inhibits cell proliferation and invasiveness in breast cancer via PI3K/Akt/mTOR signaling pathway. Onco Targets Ther 12: 10639-10650, 2019.

42. Jia LX, Zhang WM, Zhang HJ, Li TT, Wang YL, Qin YW, Gu H and Du J: Mechanical stretch-induced endoplasmic reticulum stress, apoptosis and inflammation contribute to thoracic aortic aneurysm and dissection. J Pathol 236: 373-383, 2015.

43. Marti HH and Risau W: Angiogenesis in ischemic disease. Thromb Haemost 82 (Suppl 1): S44-S52, 1999.

44. Rapisarda A and Melillo G: Role of the VEGF/VEGFR axis in cancer biology and therapy. Adv Cancer Res 114: 237-267, 2012.

45. Hoeben A, Landuyt B, Highley MS, Wildiers H, Van Oosterom AT and De Bruijn EA: Vascular endothelial growth factor and angiogenesis. Pharmacol Rev 56: 549-580, 2004.

46. Bastiaansen AJ, Ewing MM, de Boer HC, van der Pouw Kraan TC, de Vries MR, Peters EA, Welten SM, Arens R, Moore SM, et al: Lysine acetyltransferase PCAF is a key regulator of arteriogenesis. Arterioscler Thromb Vasc Biol 33: 1902-1910, 2013.

47. Dellinger MT and Brekken RA: Phosphorylation of Akt and ERK1/2 is required for VEGF-A/VEGFR2-induced proliferation and migration of lymphatic endothelium. PLoS One 6: e28947, 2011.

48. Yang GY, Yao JS, Huey M, Hashimoto T and Young WL: Participation of PI3K and ERK1/2 pathways are required for human brain vascular smooth muscle cell migration. Neurochem Int 44: 441-446, 2004

49. Shao Y, Li G, Huang S, Li Z, Qiao B, Chen D, Li Y, Liu H, Du J and Li P: Effects of extracellular matrix softening on vascular smooth muscle cell dysfunction. Cardiovasc Toxicol 20: 548-556, 2020.

50. Moncayo-Arlandi J, López-García A, Fernández MC, Durán AC and Fernández B: Osteoglycin deficiency does not affect atherosclerosis in mice. Atherosclerosis 237: 418-425, 2014.

51. Lee KP, Sudjarwo GW, Jung SH, Lee D, Lee DY, Lee GB, Baek S, Kim DY, Lee HM, Kim B, et al: Carvacrol inhibits atherosclerotic neointima formation by downregulating reactive oxygen species production in vascular smooth muscle cells. Atherosclerosis 240: 367-373, 2015.

52. Li HY, Leu YL, Wu YC and Wang SH: Melatonin inhibit in vitro smooth muscle cell inflammation and proliferation and atherosclerosis in apolipoprotein E-deficient mice. J Agric Food Chem 67: 1889-1901, 2019. 